\title{
Convolutional neural networks for segmenting xylem vessels in stained cross-sectional images
}

\author{
Angel Garcia-Pedrero' ${ }^{1}$ (1) Ana I. García-Cervigón ${ }^{2} \cdot$ José M. Olano $^{1} \cdot$ Miguel García-Hidalgo ${ }^{1}$ • \\ Mario Lillo-Saavedra ${ }^{3} \cdot$ Consuelo Gonzalo-Martín $^{4} \cdot$ Cristina Caetano $^{2} \cdot$ Saúl Calderón-Ramírez $^{5}$
}

Received: 16 April 2019/Accepted: 5 October 2019/Published online: 24 October 2019

(C) Springer-Verlag London Ltd., part of Springer Nature 2019

\begin{abstract}
Xylem is a vascular tissue that conducts sap (water and dissolved minerals) from the roots to the rest of the plant while providing physical support and resources. Sap is conducted within dead hollow cells (called vessels in flowering plants) arranged to form long pipes. Once formed, vessels do not change their structure and last from years to millennia. Vessels' configuration (size, abundance, and spatial pattern) constitutes a record of the plant-environment relationship, and therefore, a tool for monitoring responses at the plant and ecosystem level. This information can be extracted through quantitative anatomy; however, the effort to identify and measure hundreds of thousands of conductive cells is an inconvenience to the progress needed to have solid assessments of the anatomical-environment relationship. In this paper, we propose an automatic methodology based on convolutional neural networks to segment xylem vessels. It includes a post-processing stage based on the use of redundant information to improve the performance of the outcome and make it useful in different sample configurations. Three different neural networks were tested obtaining similar results (pixel accuracy about $90 \%$ ), which indicates that the methodology can be effectively used for segmentation of xylem vessels into images with non-homogeneous variations of illumination. The development of accurate automatic tools using CNNs would reduce the entry barriers associated with quantitative xylem anatomy expanding the use of this technique by the scientific community.
\end{abstract}

Keywords Convolutional neural networks $\cdot$ Dendrology $\cdot$ Xylem cells $\cdot$ Image segmentation

\section{Introduction}

Forests perform a dominant role in terrestrial carbon cycle, being a major carbon reservoir and playing a role as both carbon sink and source [1]. Forests net contribution to $\mathrm{CO}_{2}$

Angel Garcia-Pedrero

angelmario.garcia@uva.es

EiFAB-iUFOR, Universidad de Valladolid, Soria, Spain

2 Departamento de Biologìa, Universidad de Cádiz, Puerto Real, Spain

3 Faculty of Agricultural Engineering, University of Concepción, Chillán, Chile

4 Department of Computer Architecture and Technology, Universidad Politécnica de Madrid, Madrid, Spain

5 Pattern Recognition and Machine Learning Group, Instituto Tecnológico de Costa Rica, Cartago, Costa Rica atmospheric levels depends on forest cover changes as well as on tree growth rates. Enhanced $\mathrm{CO}_{2}$ levels may fuel higher photosynthetic rates, whereas higher mean temperatures may have a positive effect by extending growth season length [2], particularly in temperate and boreal forests. Nevertheless, an increase in drought frequency and intensity associated with higher temperatures and higher climate variability may reduce tree growth and even lead to forest dieback [3]. Thus, the future contribution of forest systems to carbon budget requires deciphering their response to ongoing climate change.

Xylem conduits connect roots and photosynthetic apparatus sustaining the flow of a continuous water column. Xylem conduits' anatomical configuration reflects adjustments to change in hydric conditions from monthly to decadal scales [4]. It has been commonly assessed that conductive cells face a dilemma between efficiency (higher water flow) and safety (reduced embolism risk), so that 
traits related to higher efficiency as higher diameter cell area are at odds with flow safety [5]. This paradigm is currently under debate since adjustment in xylem features as conductive tissue density might create both safe and efficient xylem configurations [6, 7]. Since xylem is dominated by dead cells, resistant to decay, and lasting potentially from decades to millennia [8], it constitutes a permanent record of tree-environment relationship [9]. Moreover, in areas under seasonal climatic or environmental conditions, xylem is produced at annual pulses of growth separated by different anatomical features [10] and that can be related unequivocally to calendar years.

Xylem conductive cell quantitative anatomy is a promising field for a better comprehension of plant response to environment. Nevertheless, the tedious work of identifying and measuring hundreds of thousands of conductive cells hampers the necessary progress to have robust assessments of anatomy-environment relationship. Large advances have been done in physical procedures to optimize the achievement of high-quality wood section and digital image acquisition [11]; however, the identification of individual conductive cells still requires a thorough human supervision.

Nowadays, various computer programs are used to analyze wood images and identify wood vessel lumina. Among them, ROXAS [12] is the most widely used. ROXAS is an automatic tool based on traditional image processing methods that works on Image Pro Plus. ${ }^{1}$ In general, these tools are very sensitive to sample processing and image quality and require the tuning of different parameters related to color and vessel shape and size. Even after careful sample preparation, vessels may break in some parts of the sample, or some dye stains may remain. Moreover, images may generally have irregular lighting, uneven spots, and blurred regions. These problems produce low-quality areas which then cause error during the segmentation of vessels. To date, the way to correct these flaws is a manual editing process that can take a certain amount of time depending on the sample quality. This manual editing process can become an endless task when a given study needs to analyze dozens or even hundreds of images. Therefore, obtaining accurate segmentations can greatly improve automatic image analysis, reducing the amount of time spent on manual editing.

Although automatic and semi-automatic cell segmentation has been widely studied in the biomedical field [13], xylem cell segmentation has been less studied. Few papers can be found in the literature, most of which use a combination of several classic image processing techniques [14] such as thresholding and mathematical morphology. In

\footnotetext{
${ }^{1}$ http://www.mediacy.com/imageproplus. Last accessed on April 2009.
}

[15], the authors present a tool for analyzing tree rings by segmenting vessels using the Otsu's thresholding algorithm. Opening and closing morphological operations are used in conjunction with the watershed algorithm to eliminate minor disturbances and achieve a general smoothing of the vessel edges. Similar approaches are followed by $[16,17]$ to segment the walls of conductive cells and the cells of tree roots, respectively. Works such as $[18,19]$ explore the use of genetic algorithms for selecting the optimal threshold to segment wood cells. In [20], Canny detection edge operator is used to find high and low thresholds. However, these grayscale threshold-based methods tend to fail in cases where images have highly variable color dynamics. In general, some post-processing steps (eg. edge detection [21] and active contours [5, 22]) are required to refine segmented vessels. Approaches based on mathematical morphology segmentation use operations such as dilation, erosion, opening, and closing for segmenting wood vessels (e.g., [23-25]). Other method from mathematical morphology used in the literature is the watershed segmentation (e.g., [15, 26, 27]). Since approaches based on this method tend to over-segment vessels, they require pre-processing (e.g., filtering operations [28, 29]) and post-processing operations (e.g., merging regions [30]). Other works use more sophisticated methods for segmenting wood vessels, such as level sets based on Mumford-Shah model [31], gradient vector flow snake [32]. In general, these methods tend to have problems with low contrast areas, as well as being very sensitive to the initial position of the level set function that makes them prone to converge to false edges.

Clustering techniques are also used to segment xylem conduits, but instead of working directly on image pixels, they extract image features through processes such as gray level concurrency matrix (GLCM) [33] and multiscale morphology [34]. These methods fail when the vessels show variations in intensity or orientation, since the extracted features do not take into account such cases.

Recently, supervised learning approaches have been used to segment images [35, 36]. In particular, convolutional neural networks (CNNs) have demonstrated a remarkable performance for segmenting cells in biomedical microscope images [37-41]. CNNs are variants of neural feedback networks inspired by the visual cortex of mammals, with layers, known as convolutional layers. These layers perform local processing of contiguous features within each layer, acting as a nonlinear feature detector [42]. CNNs exploit the local spatial correlation present in an image by generating a pattern of local connectivity between neurons in adjacent layers which produces high-level features as it approaches the output layer. Unlike traditional supervised learning methods where 
feature extraction and classification are learned separately, CNNs learn these steps together.

Despite their benefits in image segmentation, to the best of our knowledge, CNNs are just beginning their journey into dendrochronological applications. In [43], the authors present a CNN approach to segment xylem vessels from microscopic images. The approach is based on a fully convolutional network, called Unet [41], trained from 23 images. Since the images are larger than the GPU's memory, they are divided into patches for training. Once the models have been trained, during the test phase, the images are processed using a tile overlay strategy that results in a complete segmentation of the images. Despite the small number of images, the results shown are superior to those obtained using techniques such as thresholding and level sets.

This paper is an extended version of work published in [43]. We extended our previous work by considerably increasing the number of images to be studied, and introducing during the prediction phase an improved version of the tile strategy includes test time augmentation (TTA) [44] on three convolutional neural networks. This post-processing strategy allows to exploit the redundant information produced by several transformations of the same region providing greater robustness to the segmentation of xylem vessels. The objective of this work is to provide a robust $\mathrm{CNN}$ approach for segmenting xylem cells and to investigate the performance of the post-processing strategy by comparing the results using different deep architectures and diverse parameters. The remainder paper is structured as follows. In Sect. 2, the data used are presented, while Sect. 3 describes the methodology. In Sect. 4, the results are discussed to end with some conclusions and future works in Sect. 5.

\section{Materials}

Nothofagus pumilio (Poepp. et Endl.) Krasser (Nothofagaceae) is a deciduous tree species native to the southern Andes of Chile and Argentina. It extends through a wide latitudinal range, covering a climatic gradient that ranges from Mediterranean to cold temperate areas $\left(35^{\circ}-56^{\circ}\right.$ south latitude). In this species, vessels' arrangement is diffuse porous with a slight tendency to semi-ring porosity [45], i.e., vessels show approximately the same size all along the ring, although they tend to narrow as approaching the ring boundary.

In this study, we used branch sections of approximately $2 \mathrm{~cm}$ diameter belonging to 23 different Nothofagus pumilio individuals. These samples were collected to be used in a previous study in which xylem adjustments of Nothofagus pumilio were evaluated under a wide range of climatic conditions [7]. Samples were collected in five sites covering nearly the whole latitudinal range of the species in Chile. At each site, samples were taken at four different elevations corresponding to different forest structures. From lowest to highest, the four elevations corresponded to closed and mature forests, intermediate stands between closed forests and timberline, timberline, and treeline. The treeline was defined as the uppermost limit of individual trees with an upright growth form of at least $3 \mathrm{~m}$ [46]. Wood samples were processed with a sledge microtome [47] obtaining $10 \mu \mathrm{m}$ width transversal sections from each of the 23 branch samples. Afterward, sections were stained with a mix of alcian blue/acetic acid (1\%) and safranin/ ethanol (1\%) and gradually dehydrated by increasing concentrations of ethanol solutions. Finally, dehydrated samples were washed by xylol and preserved with Eukitt glue and a coverslip. Mounted and stained sections were acquired as RGB images with $\times 40$ magnification using a Nikon D90 digital camera adapted to a Nikon Eclipse 50i microscope. Due to magnification level, multiple images were took to cover the whole cross section from pith to bark and merged using PTGUI v8.3.10 Pro. ${ }^{2}$ Each merged image had an average size of $2210 \times 1248$ pixels.

Cross-sectional images were segmented using ROXAS v3.0 [8, 12]. For this purpose, different parameter settings were created depending on the variability found in image quality. Thus, by means of visual inspection the maximum and minimum lumen area, the shape of the vessels and various color parameters were adjusted. These settings were used to automatically segment all samples, and the automatic outputs were corrected manually until satisfactory results were obtained. During this manual editing process, the boundaries of the rings were first drawn. Then, erroneously segmented vessels (e.g., ray parenchyma) were removed and other erroneous identifications were rectified. Exclusion areas were used to leave out lower-quality parts of the image (e.g., dye stains or vessels broken during sample preparation). In the final stage of manual editing, the 100 largest vessels per image were checked to split those that were erroneously merged during automatic analysis due to the low contrast between the vessels. This error search was performed until no correction was necessary, on average after verifying 20 consecutive vessels. Therefore, there may be some areas that have not been corrected. We spend an average of 30 min manually editing each sample. An example of an image where vessels have been manually edited is shown in Fig. 1; for visualization purposes, only a small part of the cross-sectional image and its ground truth are displayed enlarged.

\footnotetext{
${ }^{2}$ https://www.ptgui.com. Last accessed on March 2019.
} 


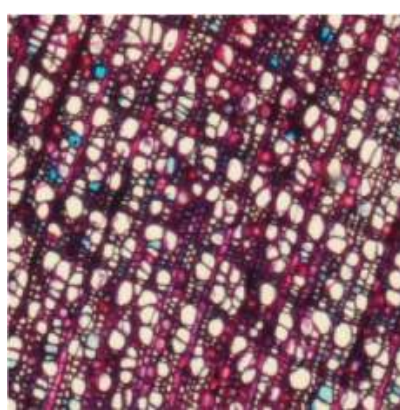

(a) Cross-sectional image

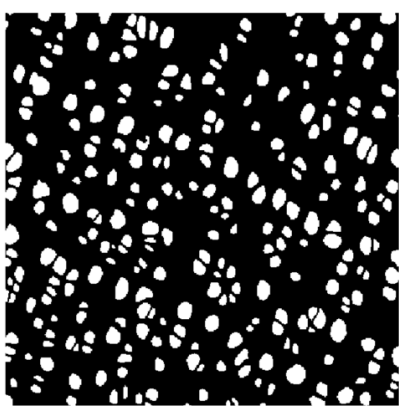

(b) Ground-truth
Fig. 1 Example of a cross-sectional image (a) and its corresponding ground truth (b)

\section{Methodology}

Our workflow consists of two parts (Fig. 2): (1) a CNN trained with an augmented set of patches extracted from the training cross-sectional images, (2) the trained model is used to provide the segmentation of an image considerably larger than the size of the patches with which it was trained. To do this, the image is broken down into patches, from which predictions are generated for each pixel. Overlap and TTA operations allow to have a redundancy of the predictions providing a greater robustness during the estimation of the pixel class. This information is integrated by means of a soft vote system. The main points of the methodology are described below.

\subsection{Data pre-processing}

Cross-sectional images were cropped into overlapped patches of 224 by 224 pixels. Hereinafter these patches are referred to as data patches. Considering that there are parts of the image that are not included from the study, only data patches whose area is covered by at least $20 \%$ of vessels were considered during training. To avoid overfitting during training phase, data augmentation was performed applying to each data patch a Dihedral [48] random transformation from Dih4 group (Fig. 3). In addition, with a probability of 0.5 , a random rotation with grades of -5 to 5 was also performed.

GT masks were generated from annotations saved in a format compatible with ROXAS software. To do this, the annotations were first converted into images by assigning a unique label to the surface of each vessel. The assigned labels ranged from 1 to $N$, while the background was assigned the 0 label. From them, three classes were generated corresponding to the background, the vessel, and its outline. The masks were encoded using a one-hot encoding scheme. In such a way that each pixel had representation of a vector of zeros, being the target class labeled with the value one. These masks were subjected to the same processes as the data patches, so the segmented regions matched the vessels.

\subsection{CNN models}

For segmenting xylem vessels, we used three state-of-theart neural network models: Unet [41], Linknet [49], and Feature Pyramid Network (FPN) [50]. The architectures of these networks are shown in Figs. 4, 5, and 6. These networks can be broadly described as multiscale decodercoders models with skip connections based on CNNs. The encoding part extracts features from the data while reduces the width and height of the input data. On the other hand, the decoder part upsamples feature maps while it halves the number of feature channels. Skip connections alleviate the vanishing gradient problem [51] improving the learning process. Finally, the output layer uses an $1 \times 1$ convolution along with an activation function to map the feature vector to an output matrix with the width and height of the input image and the same number of channels as classes. In this work, softmax function [52] was used as activation function.

The main difference between these three neural network models lies in the way the coding path information is integrated into the decoding part. Thus, while the U-net architecture (Fig. 4) concatenates a map of characteristics of each of the downsampling routes to the corresponding upsampling route, in Linknet the input of each encoder layer is added to the output of its corresponding decoder, as can be seen in Fig. 5, with the aim of recovering the spatial information lost during the downsampling process. On the other hand, the FPN includes a top-down route and a bottom-up route with side connections. Although from the implementation point of view, this architecture can be interpreted in a similar way to the U-net and Linknet architectures (with a block of encoders and decoders), the main difference is that the lateral connections merge feature maps of the same spatial size, from encoder path to the output of each corresponding decoder but also this information is bypassed to the interpolation block layers, located between the decoder block and the output layer (Fig. 6).

Similar to [53], the encoders of all the networks were built using a Resnet34 [51] encoder pretrained on ImageNet. The use of trained weights on ImageNet data was preferred over training from scratch because it usually accelerates convergence and helps reduce overfitting on small data sets [54]. In this regard, the encoder block is equivalent to a residual block [51]. As can be seen in (Fig. 7), the encoder block consists of two $3 \times 3$ convolutional layers with the same number of output channels, stride 1 . The input to each convolutional layer is preceded by a batch normalization layer (BN), a ReLU activation 


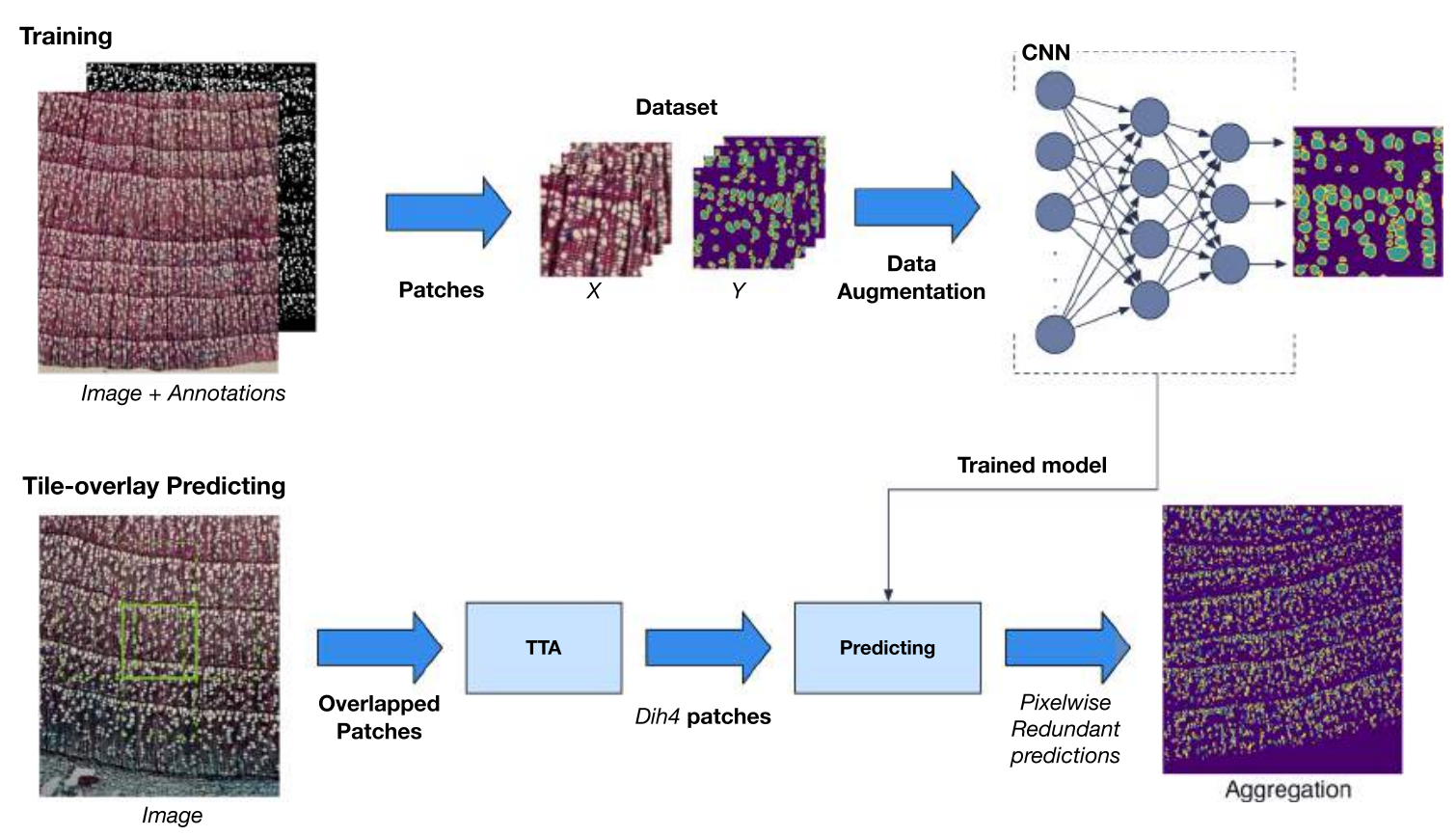

Fig. 2 Overview of our workflow for segmenting xylem vessels

Fig. 3 Transformations of the Dih4 group on a data patch. Rot $_{90}, \operatorname{Rot}_{180}$, and Rot $_{270}$ indicate $90^{\circ}, 180^{\circ}$, and $270^{\circ}$ counterclockwise rotations, respectively. Refl $_{h}$ and Refl $_{v}$ indicate horizontal and vertical reflections, respectively

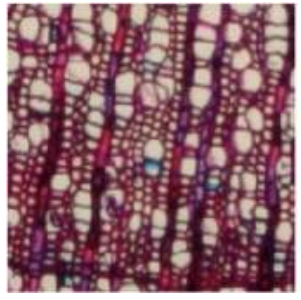

(a) Identity

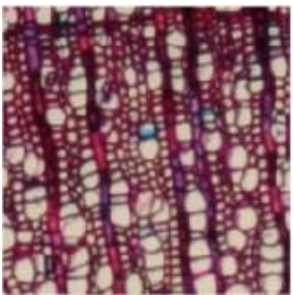

(e) $\operatorname{Re} f l_{v}$

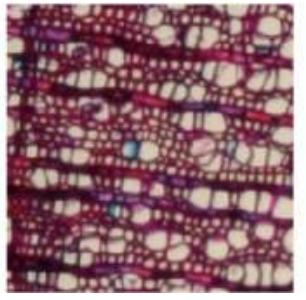

(b) $\operatorname{Rot}_{90}$

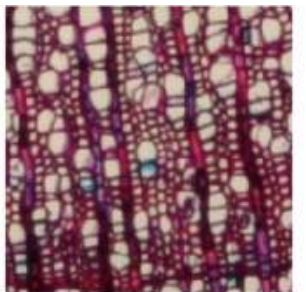

(f) $\operatorname{Refl} l_{h}$

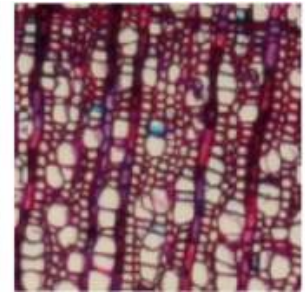

(c) $\operatorname{Rot}_{180}$

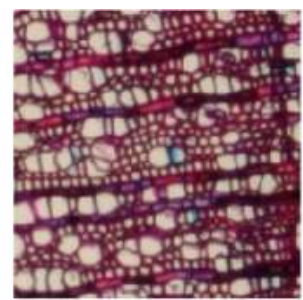

(g) $\operatorname{Rot}_{90}+R e f l_{h}$

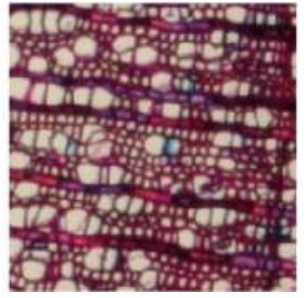

(d) $\operatorname{Rot}_{270}$

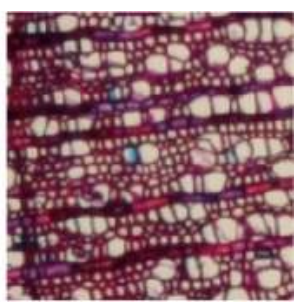

(h) $\operatorname{Rot}_{270}+R e f l_{h}$ function (AF), and a zero padding operation (ZP). BN layer normalizes the activations of the previous layer in each batch, by applying a data standardization (mean zero and standard deviation close to one). ZP layer pads the input with zeros, adding two elements to the width and height. The ReLU function rectifies the input signal by removing values less than zero. A skip connection adds the output of a previous block to the output of the current block. Each decoder block reduces the width and height of the input reduced by half.

On the other hand, the decoder block (Fig. 8) includes three $3 \times 3$ convolutional layers followed by $\mathrm{BN}$ and $\mathrm{ZP}$ layers. The upsampling layer repeats the rows and columns of the input data doubling in size. Unlike the encoder block, the decoder increases the width and height of the input by two, while reducing the number of channels.

Finally, the interpolation block (Fig. 9) of the FPN architecture is similar to the decoder block with the exception that the input is interpolated using the algorithm of the nearest neighbors, increasing its height and width by a factor $f$.

A detailed description of the each layer can be found in [55].

Networks were re-trained using the Adam optimizer with learning rate of $1 \mathrm{e}^{-3}$ and a batch size of 32. Each 


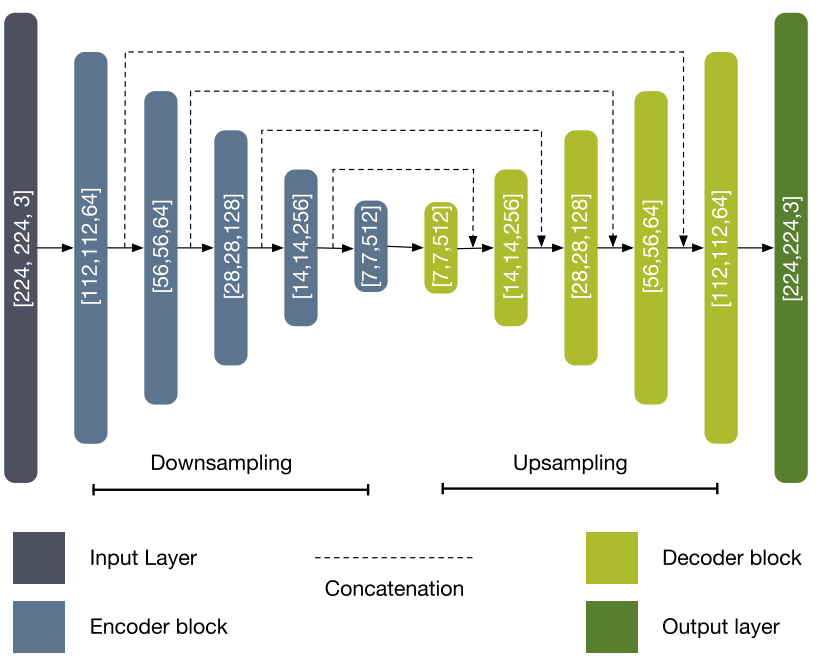

Fig. 4 Unet architecture. The numbers in brackets represent the outputs of the blocks, in the format [height, width, number of channels]

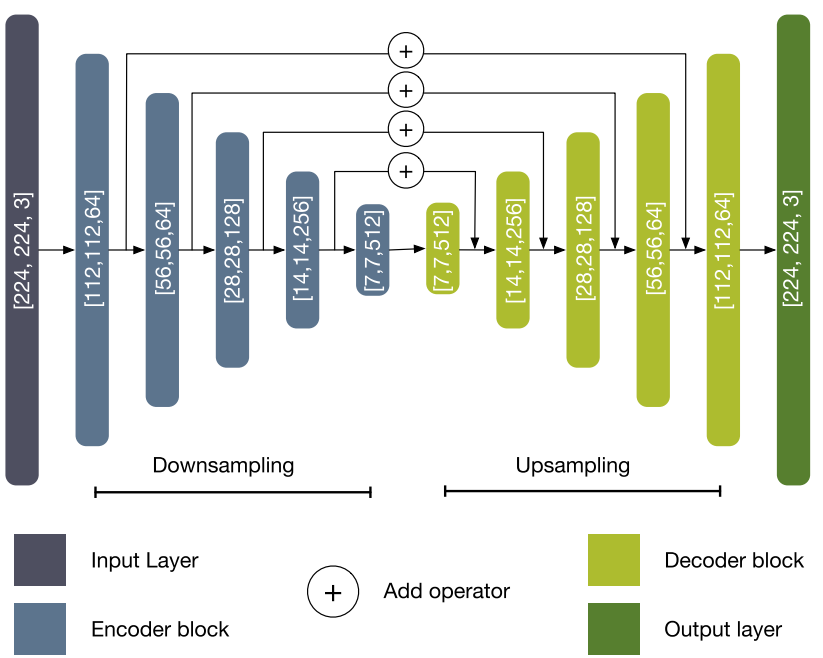

Fig. 5 Linknet architecture. The numbers in squared brackets indicate the outputs of the blocks [height, width, number of channels]

model was trained during 20 epochs, with approximately $90 \mathrm{~K}$ data patches used for training and $10 \mathrm{~K}$ patches for validation (model calibration).

To train the CNNs, we used a combination of categorical cross-entropy [56] and Jaccard distance [57] as the loss function, which is given as:

$$
\begin{gathered}
\mathcal{L}(Y, \hat{Y})=-\frac{1}{N} \sum_{i}^{N} \sum_{c}^{C} y_{i, c} \log _{2} \hat{y}_{i, c} \\
-\frac{\sum_{i}^{N} \sum_{c}^{C} \hat{y}_{i} y_{i}+\epsilon}{\sum_{i}^{N} \sum_{c}^{C}\left(\hat{y}_{i, c}+y_{i, c}-\hat{y}_{i, c} y_{i, c}\right)+\epsilon}+1
\end{gathered}
$$

where $y_{i, c} \in Y$ and $\hat{y}_{i, c} \in \hat{Y}$ denote the ground truths and the predicted probabilities of $i$ th pixel image for the class $c$, respectively, $N$ is the total number of pixels in the image
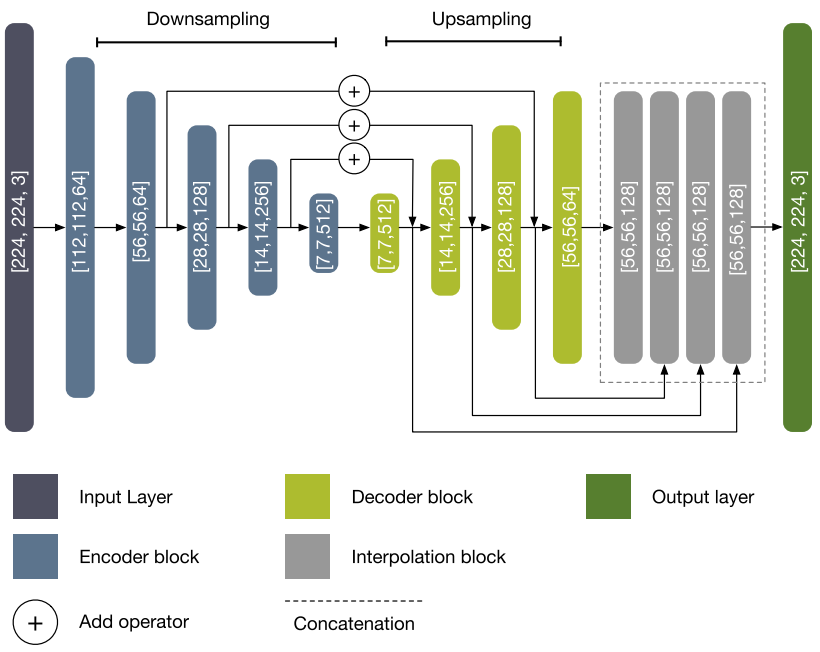

Fig. 6 FPN architecture. The outputs of each block are represented in the following format [height, width, number of channels]

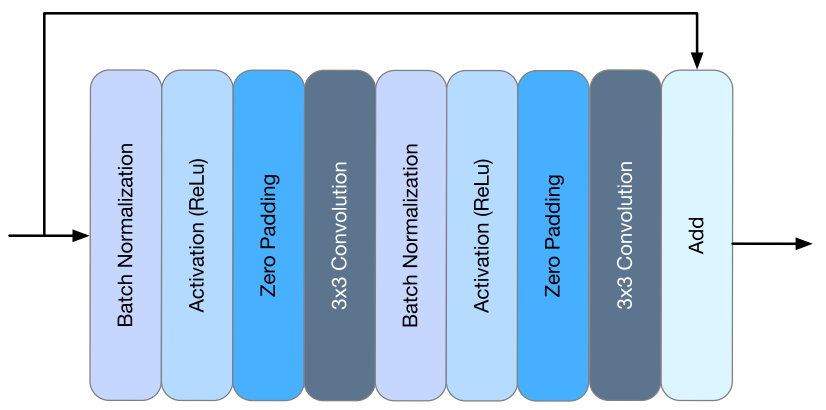

Fig. 7 Encoder block

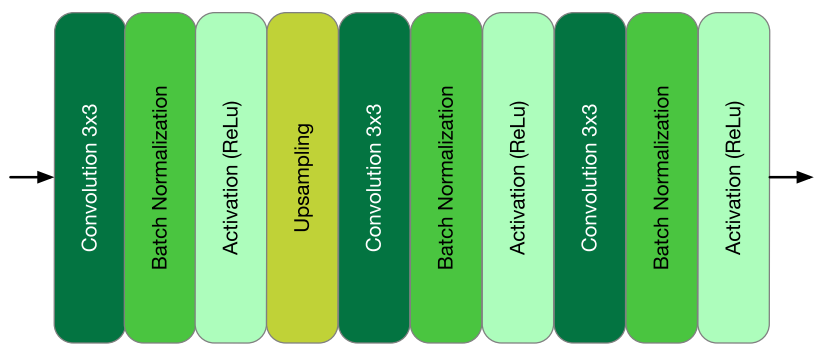

Fig. 8 Decoder block

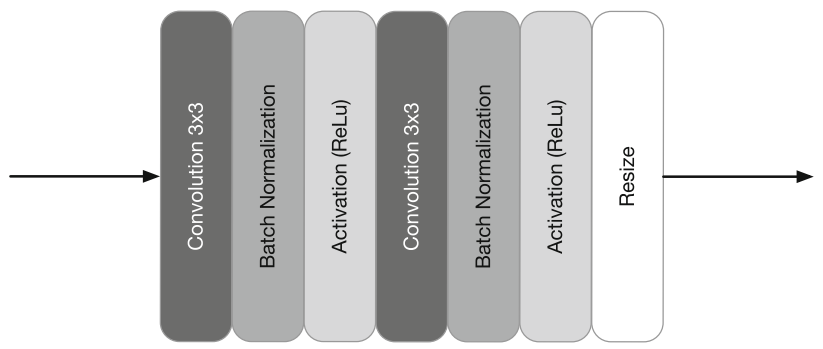

Fig. 9 Interpolation block 
$(|Y|), C$ is the total number of classes a pixel can belong to, and $\epsilon$ is a smoothing factor used to avoid zero division error. In this work, an $\epsilon=1 \mathrm{e}^{-12}$ was used.

\subsection{Post-processing}

Because the images under study are larger than the patches used for training, it is necessary to split each image into $N$ small sub-images of the same size of data patches. First, a mirror padding strategy is used to label the pixels around the boundaries of the image. Then, the resulting image is divided into several overlapping patches, from which a CNN performs the segmentation. During the prediction phase, test time augmentation (TTA) [44] is performed. Similar to what data augmentation does with the training set, the purpose of TTA is to make random transformations to the test images. Thus, instead of showing the regular images only once to the trained model, the various versions of the images are shown several times [44]. The average of the predictions of each corresponding image is considered to make the final prediction. The intuition behind this is that the transformations allow the model to have better chances to identify the target and predict accordingly. In general, different transformations can be used. In this work, we have used the eight transformations of the Dih4 group. Once the model provides the segmented images, the inverse transformation is performed on them, in such a way that all the pixels agree. The final image segmentation is given by a soft voting that returns the final class label $\overline{y_{i}}$ as arg max of the sum of predicted probabilities:

$\bar{y}_{i}=\underset{c}{\arg \max } \sum_{t}^{T} w_{c} \hat{y}_{i, c}^{t}, \quad c \in\{0,1,2\}$

where $\hat{y}_{i, c}^{m}$ is the probability of the $i$ th pixel inferred to class $c$ given by predicting the image to which the transformation $t$ was applied, $w_{c}$ is the weight. In this work, the weights $\left(w_{c}\right)$ were defined as $1 / C$.

\subsection{Experimental setup}

The performance of the proposed approach for segmenting xylem vessels was tested trough a tenfold cross-validation using the 113 available cross-sectional images. In this paper, four common indices were used to assess segmentation performance [58]: pixel accuracy (PA), mean pixel accuracy (MPA), mean intersection over union (MIoU), and frequency weighted intersection over union (FWIoU). These indices define the correspondence between the ground truth $\left(y_{i}\right)$ and the estimate made by our approach $\left(\bar{y}_{i}\right)$ in terms of $p_{i, j}$, that is, the number of pixels from class $i$ inferred to class $j$, where $p_{i, i}$ represents the number of true positives, while $p_{i, j}$ and $p_{j, i}$ can be interpreted as false positive and false negatives.

PA establishes the relationship between the number of correctly classified pixels and the total number of them.

$\mathrm{PA}=\frac{\sum_{i}^{C} p_{i, i}}{\sum_{i}^{C} \sum_{j}^{C} p_{i, j}}$.

MPA is a variation of PA where the correct proportion of pixels per class is calculated and then averaged over the total number of classes.

$\mathrm{MPA}=\frac{1}{C} \sum_{c=1}^{C} \frac{p_{i, i}}{\sum_{j=1}^{C} p_{i, j}}$.

MIoU computes a relationship between the intersection and the union of the ground truth and the obtained segmentation.

MIoU $=\frac{1}{C} \sum_{i}^{C} \frac{p_{i, i}}{\sum_{j}^{C} p_{i, j}+\sum_{j}^{C} p_{j, i}-p_{i, i}}$.

FWIoU is a modification of MIoU that weighs the importance of each class depending on its frequency of occurrence.

FWIoU $=\frac{1}{\sum_{i}^{C} \sum_{j}^{C} p_{i, j}} \sum_{i}^{C} \frac{\sum_{j}^{C} p_{i, j} p_{i, i}}{\sum_{j}^{C} p_{i, j}+\sum_{j}^{C} p_{j, i}-p_{i, i}}$.

All these metrics range from 0 to 1 , where 1 indicates a perfect correspondence between the ground truth and the segmentation.

All experiments have been done using an NVIDIA TITAN Xp GPU with 3840 CUDA cores and 12 GB of memory. Codes were developed in Python using Keras framework [55].

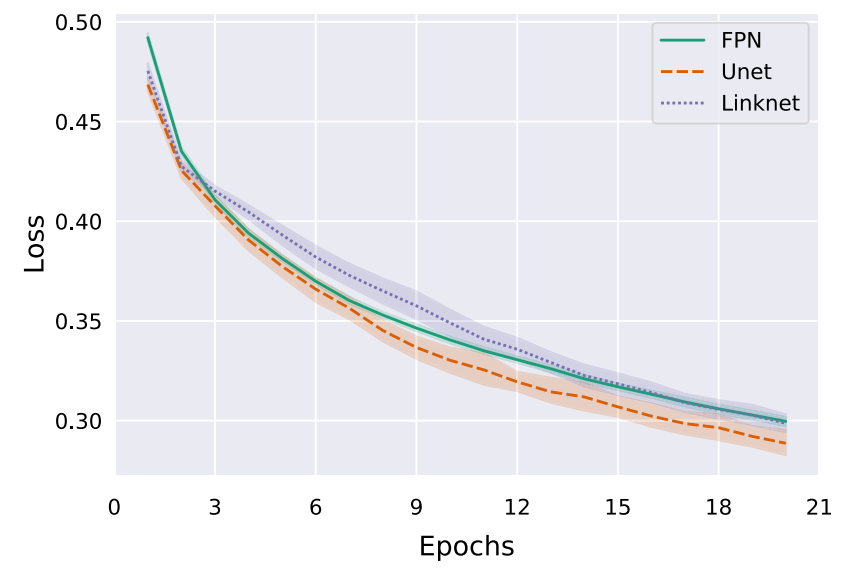

Fig. 10 Loss function results during training in the tenfolds 
Table 1 Normalized confusion matrix. Unet model without TTA and overlap $=0$

\begin{tabular}{llll}
\hline & Background & Vessel & Outlines \\
\hline Background & 0.958307 & 0.011151 & 0.030542 \\
Vessel & 0.033100 & 0.866343 & 0.100557 \\
Outlines & 0.196538 & 0.125533 & 0.677929 \\
\hline
\end{tabular}

Table 2 Normalized confusion matrix. Linknet model without TTA and overlap $=0$

\begin{tabular}{llll}
\hline & Background & Vessel & Outlines \\
\hline Background & 0.956820 & 0.011532 & 0.031647 \\
Vessel & 0.025009 & 0.881513 & 0.093477 \\
Outlines & 0.173165 & 0.134801 & 0.692034 \\
\hline
\end{tabular}

Table 3 Normalized confusion matrix. FPN model without TTA and overlap $=0$

\begin{tabular}{llll}
\hline & Background & Vessel & Outlines \\
\hline Background & 0.960007 & 0.010701 & 0.029292 \\
Vessel & 0.033864 & 0.869960 & 0.096176 \\
Outlines & 0.207142 & 0.114797 & 0.678062 \\
\hline
\end{tabular}

\section{Results and discussion}

During training, the behavior of the three networks according to the loss function (Eq. 1) was similar (see Fig. 10). Of these, Unet had a slightly better error; however, it also had a higher variability during the tenfolds, on the other hand, FPN showed a lower variability.

The normalized confusion matrices of the Unet, Linknet, and FPN segmentations (without TTA or overlap) of the tenfold test set images, shown in Tables 1, 2 and 3, indicate that the main errors occurred at the outlines of the vessels, which were mainly confused with the background (Unet $19.65 \%$, Linknet $17.31 \%$, FPN $20.71 \%$ ) and the inside of the vessels (Unet 12.53\%, Linknet 13.48\%, FPN 11.47\%). This effect may be due to the fact that the transition between the boundaries between the vessels and the background (e.g., parenchyma) is not entirely clear in all images due to the blurring and variations in illumination present in some of them.

The use of redundant information provided by the prediction strategy used slightly increased the score of the results of Unet, Linknet, and FPN models (Tables 4, 5, and 6). This is also shown in the normalized confusion matrices of the Unet, Linknet and FPN models (Tables 7, 8 and 9, respectively), that by introducing the TTA and an overlap of 0.75 , the accuracy of the predictions increased slightly in all classes. In the case of Unet, the improvements over the background, vessel, and outlines classes were $0.29,0.45 \%$, and $1.54 \%$, respectively. For Linket, the improvements of the background, vessel, and outlines classes were $0.18 \%$, $0.71 \%$, and $1.61 \%$, respectively. Finally, FPN improved by $0.40 \%, 0.99 \%$ and $2.53 \%$ in the background, vessels, and outlines classes. The more redundant information were included (overlap and TTA); the better the results obtained. This effect was particularly reflected in the edges of the vessels (Fig. 11) where the uncertainty of the neural network is generally higher. In general terms, the error measures used indicated that the performance of the three models tested was similar, being slightly better FPN than Linknet and Unet.

Although all the images were generated following the same protocol, the images analyzed show great variability with respect to lighting and color due to the difference in staining intensity. The results obtained with the three models demonstrated the robustness of the neural network models to deal with variation in image characteristics. The best cases shown in Fig. 12 exhibit the potential of the neural networks used. However, in some cases, there were segmentation errors such as those shown in Fig. 13. The
Table 4 Evaluation of the segmentation of the xylem vessels produced by Unet model and the post-processing strategy

\begin{tabular}{llllll}
\hline OL & TTA & PA & MPA & MIoU & FWIoU \\
\hline 0.00 & No & $0.9194 \pm 0.0285$ & $0.8364 \pm 0.0520$ & $0.7169 \pm 0.0626$ & $0.8661 \pm 0.0431$ \\
& Yes & $0.9221 \pm 0.0277$ & $0.8410 \pm 0.0506$ & $0.7242 \pm 0.0628$ & $0.8700 \pm 0.0419$ \\
0.25 & No & $0.9207 \pm 0.0288$ & $0.8392 \pm 0.0528$ & $0.7206 \pm 0.0642$ & $0.8681 \pm 0.0434$ \\
& Yes & $0.9229 \pm 0.0279$ & $0.8428 \pm 0.0512$ & $0.7263 \pm 0.0639$ & $0.8711 \pm 0.0422$ \\
0.50 & No & $0.9210 \pm 0.0288$ & $0.8392 \pm 0.0529$ & $0.7213 \pm 0.0644$ & $0.8685 \pm 0.0435$ \\
& Yes & $0.9231 \pm 0.0279$ & $0.8429 \pm 0.0511$ & $0.7269 \pm 0.0639$ & $0.8714 \pm 0.0422$ \\
0.75 & No & $0.9212 \pm 0.0289$ & $0.8401 \pm 0.0532$ & $0.7221 \pm 0.0649$ & $0.8689 \pm 0.0436$ \\
& Yes & $\mathbf{0 . 9 2 3 3} \pm \mathbf{0 . 0 2 8 0}$ & $\mathbf{0 . 8 4 3 4} \pm \mathbf{0 . 0 5 1 3}$ & $\mathbf{0 . 7 2 7 5} \pm \mathbf{0 . 0 6 4 3}$ & $\mathbf{0 . 8 7 1 8} \pm \mathbf{0 . 0 4 2 3}$ \\
\hline
\end{tabular}

The best results are indicated in bold type 
Table 5 Evaluation of the segmentation of the xylem vessels produced by Linknet model and the post-processing strategy

\begin{tabular}{llllll}
\hline OL & TTA & PA & MPA & MIoU & FWIoU \\
\hline 0.00 & No & $0.9161 \pm 0.0308$ & $0.8264 \pm 0.0589$ & $0.7073 \pm 0.0679$ & $0.8613 \pm 0.0476$ \\
& Yes & $0.9212 \pm 0.0290$ & $0.8369 \pm 0.0566$ & $0.7212 \pm 0.0644$ & $0.8681 \pm 0.0455$ \\
0.25 & No & $0.9174 \pm 0.0311$ & $0.8293 \pm 0.0596$ & $0.7109 \pm 0.0698$ & $0.8633 \pm 0.0479$ \\
& Yes & $0.9221 \pm 0.0291$ & $0.8394 \pm 0.0564$ & $0.7239 \pm 0.0656$ & $0.8697 \pm 0.0453$ \\
0.50 & No & $0.9179 \pm 0.0312$ & $0.8296 \pm 0.0604$ & $0.7119 \pm 0.0701$ & $0.8638 \pm 0.0482$ \\
& Yes & $0.9223 \pm 0.0291$ & $0.8392 \pm 0.0569$ & $0.7244 \pm 0.0656$ & $0.8699 \pm 0.0455$ \\
0.75 & No & $0.9183 \pm 0.0312$ & $0.8308 \pm 0.0604$ & $0.7131 \pm 0.0708$ & $0.8645 \pm 0.0482$ \\
& Yes & $\mathbf{0 . 9 2 2 6} \pm \mathbf{0 . 0 2 9 2}$ & $\mathbf{0 . 8 4 0 1} \pm \mathbf{0 . 0 5 7 0}$ & $\mathbf{0 . 7 2 5 3} \pm \mathbf{0 . 0 6 6 2}$ & $\mathbf{0 . 8 7 0 4} \pm \mathbf{0 . 0 4 5 5}$ \\
\hline
\end{tabular}

The best results are indicated in bold type

\begin{tabular}{llllll}
\hline OL & TTA & PA & MPA & MIoU & FWIoU \\
\hline 0.00 & No & $0.9189 \pm 0.0296$ & $0.8316 \pm 0.0562$ & $0.7160 \pm 0.0635$ & $0.8646 \pm 0.0458$ \\
& Yes & $0.9224 \pm 0.0277$ & $0.8391 \pm 0.0539$ & $0.7255 \pm 0.0611$ & $0.8696 \pm 0.0431$ \\
0.25 & No & $0.9215 \pm 0.0293$ & $0.8377 \pm 0.0574$ & $0.7232 \pm 0.0657$ & $0.8684 \pm 0.0452$ \\
& Yes & $0.9246 \pm 0.0275$ & $0.8444 \pm 0.0549$ & $0.7316 \pm 0.0635$ & $0.8729 \pm 0.0426$ \\
0.50 & No & $0.9238 \pm 0.0292$ & $0.8417 \pm 0.0586$ & $0.7297 \pm 0.0678$ & $0.8718 \pm 0.0451$ \\
& Yes & $0.9264 \pm 0.0276$ & $0.8474 \pm 0.0563$ & $0.7367 \pm 0.0654$ & $0.8754 \pm 0.0427$ \\
0.75 & No & $0.9246 \pm 0.0293$ & $0.8441 \pm 0.0591$ & $0.7322 \pm 0.0689$ & $0.8731 \pm 0.0451$ \\
& Yes & $\mathbf{0 . 9 2 7 0} \pm \mathbf{0 . 0 2 7 5}$ & $\mathbf{0 . 8 4 9 1} \pm \mathbf{0 . 0 5 6 6}$ & $\mathbf{0 . 7 3 8 6} \pm \mathbf{0 . 0 6 6 1}$ & $\mathbf{0 . 8 7 6 5} \pm \mathbf{0 . 0 4 2 6}$
\end{tabular}

The best results are indicated in bold type
Table 6 Evaluation of the segmentation of the xylem vessels produced by FPN model and the post-processing strategy
Table 7 Normalized confusion matrix. Unet model with TTA and overlap $=0.75$

\begin{tabular}{llll}
\hline & Background & Vessel & Outlines \\
\hline Background & 0.961265 & 0.010681 & 0.028053 \\
Vessel & 0.032384 & 0.870929 & 0.096688 \\
Outlines & 0.191274 & 0.115368 & 0.693359 \\
\hline
\end{tabular}

Table 8 Normalized confusion matrix. Linknet model with TTA and overlap $=0.75$

\begin{tabular}{llll}
\hline & Background & Vessel & Outlines \\
\hline Background & 0.958638 & 0.011305 & 0.030056 \\
Vessel & 0.021967 & 0.888638 & 0.089395 \\
Outlines & 0.163498 & 0.128340 & 0.708162 \\
\hline
\end{tabular}

main failures occurred in regions discarded during manual correction such as broken or stained vessels. The automatic detection of these areas and their subsequent elimination would reduce the errors obtained. Despite this, segmentation results can serve as the initial result of a manual correction process. Since the trained models do not have to be parameterized, this is an advantage over existing software [8] that requires calibration of various parameters, reducing the time spent.

\section{Conclusions}

In this work, we addressed the problem of xylem segmentation through the use of convolutional neural networks. We introduced the use of the test time augmentation (TTA) during vessel segmentation, leading to slight improvements in the results, especially regarding vessels edges. TTA eases the work with cross sections where vessels may show different angles along the image. Our results demonstrate the potential of convolutional neural networks to effectively achieve the segmentation of the xylem vessels, overcoming aspects such as the non-

Table 9 Normalized confusion matrix. FPN model with TTA and overlap $=0.75$

\begin{tabular}{llll}
\hline & Background & Vessel & Outlines \\
\hline Background & 0.964015 & 0.010371 & 0.025614 \\
Vessel & 0.028481 & 0.879865 & 0.091654 \\
Outlines & 0.196128 & 0.100422 & 0.703450 \\
\hline
\end{tabular}


Fig. 11 Effect of the postprocessing strategy.

d (overlapping $=0.75$ and TTA) and $\mathbf{a}$ (overlapping $=0.00$ and no TTA) show predictions with and without postprocessing, respectively. b, e show in red the difference between segmentation a, $\mathbf{d}$ and their corresponding GT (c). While $\mathbf{f}$ shows the difference between a, d (color figure online)

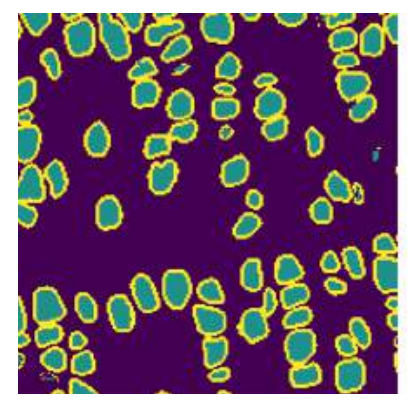

(a) $\mathrm{TTA}=\mathrm{no}, \mathrm{OL}=0.00$

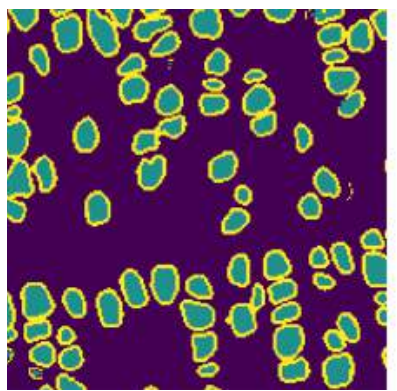

(d) $\mathrm{TTA}=$ yes, $\mathrm{OL}=0.75$

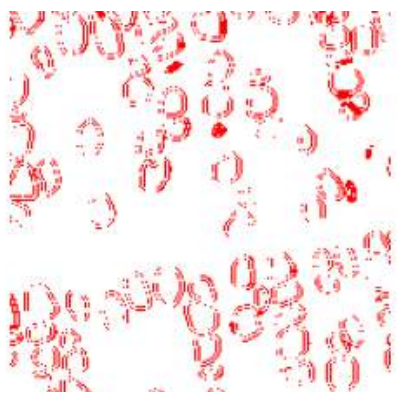

(b) Diff. (a) and (c)

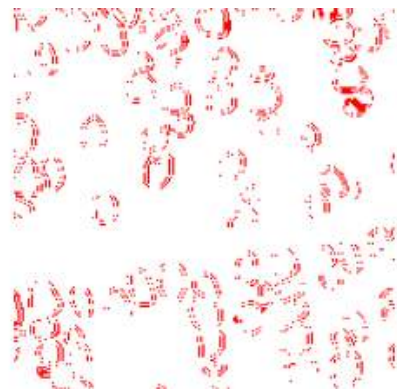

(e) Diff. (d) and (c)

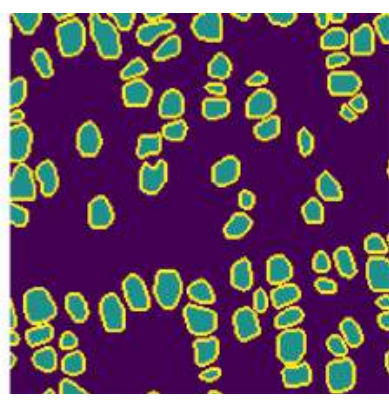

(c) $\mathrm{GT}$

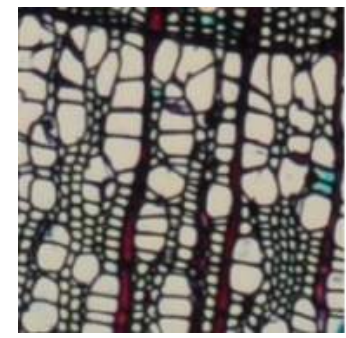

(a) Image

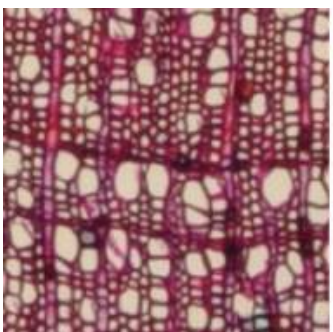

(f) Image

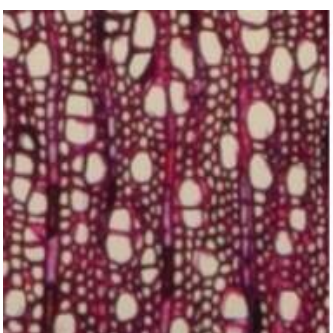

(k) Image

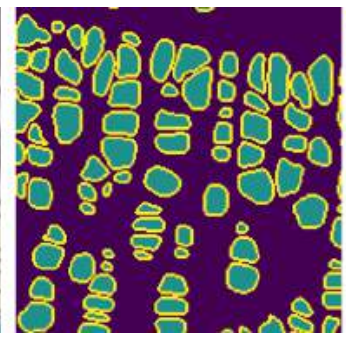

(b) GT

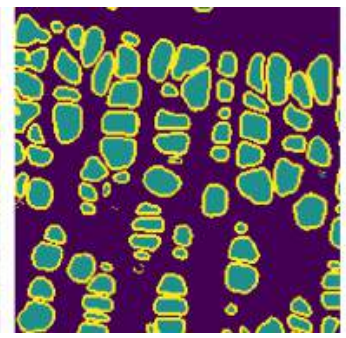

(c) Unet

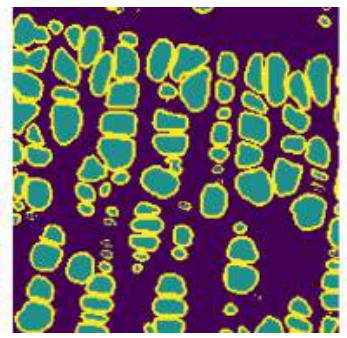

(d) Linknet

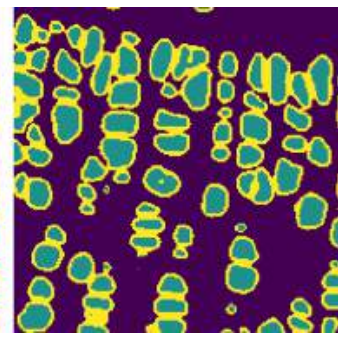

(e) FPN

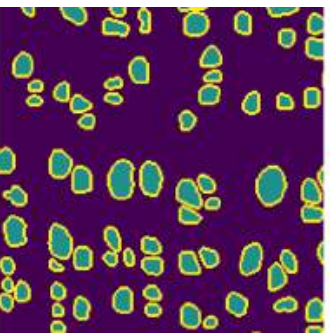

(g) GT

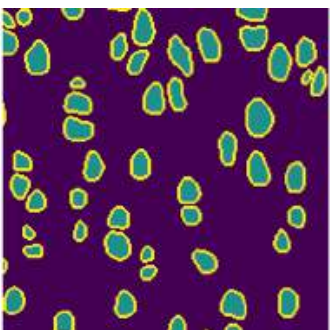

(l) GT

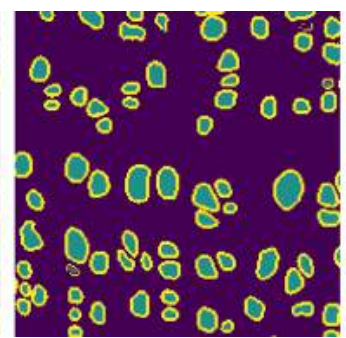

(h) Unet

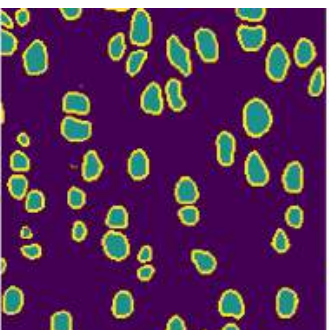

(m) Unet

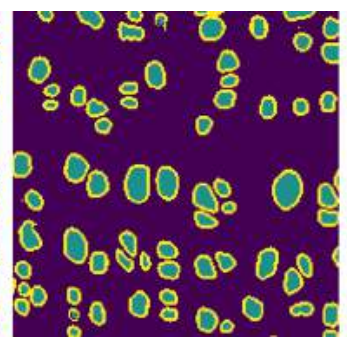

(i) Linknet

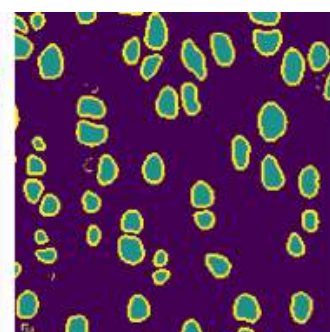

(n) Linknet

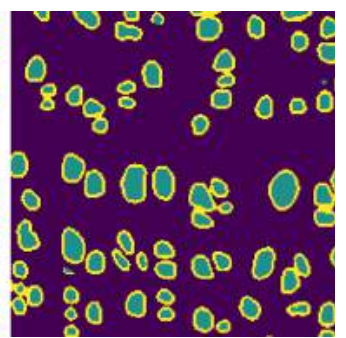

(j) $\mathrm{FPN}$

Fig. 12 Examples of the best results obtained by the models with post-processing (overlapping $=0.75$ and TTA). Each row represents the best segmented image by Unet (row 1), Linknet (row 2) and FPN (row 3). The columns 3, 4, and 5 show the performance of the models on that image 


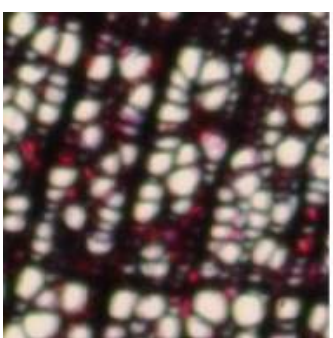

(a) Image

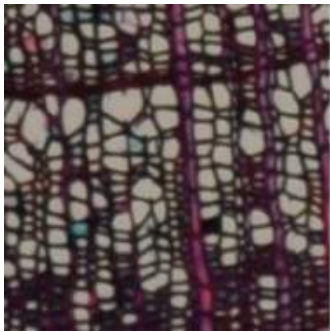

(f) Image

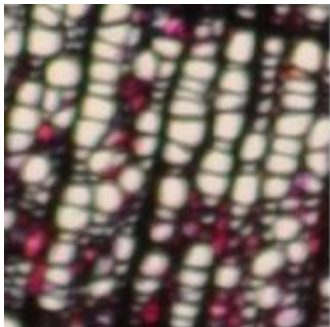

(k) Image

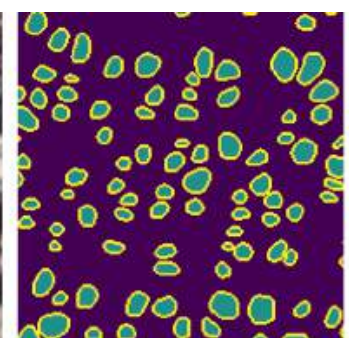

(b) GT

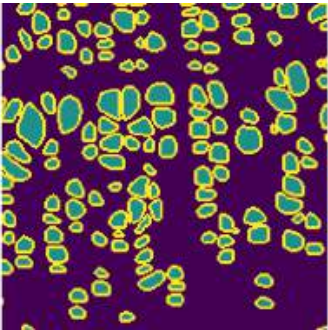

(g) GT

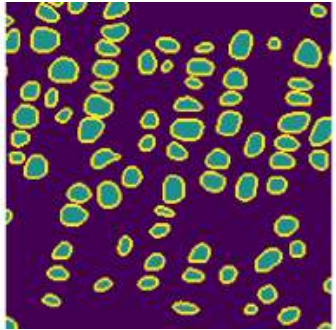

(l) GT

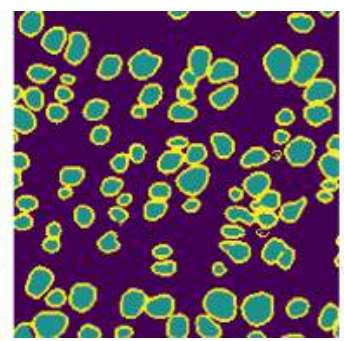

(c) Unet

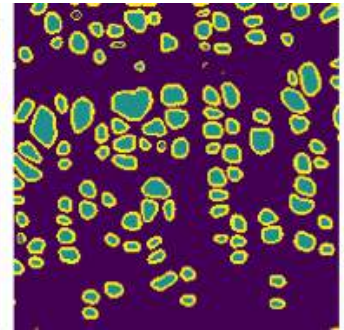

(h) Unet

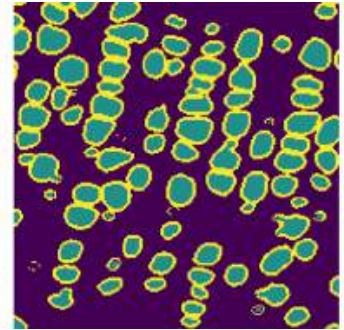

(m) Unet

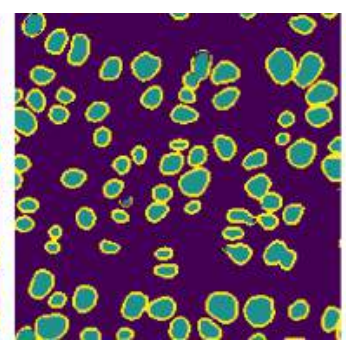

(d) Linknet

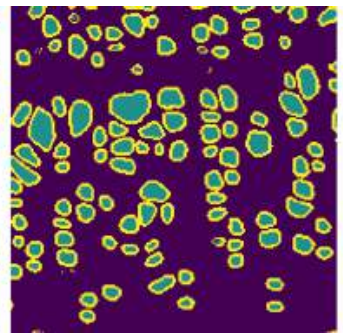

(i) Linknet

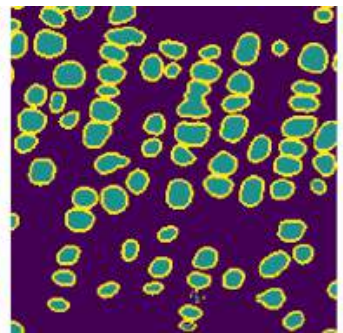

(n) Linknet

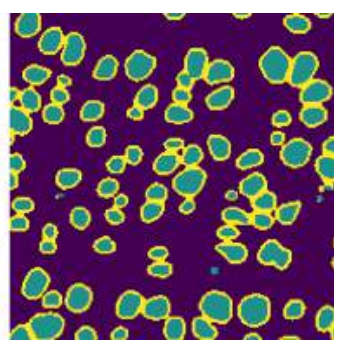

(e) $\mathrm{FPN}$

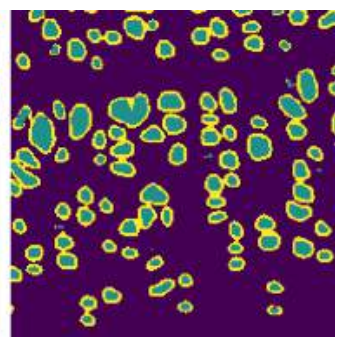

(j) $\mathrm{FPN}$

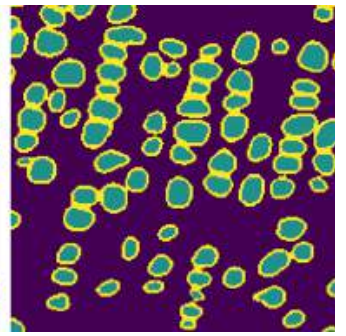

(o) FPN

Fig. 13 Examples of the worst results obtained by the models with post-processing (overlapping $=0.75$ and TTA). Each row represents the worst segmented image by Unet (row 1), Linknet (row 2) and FPN (row 3). The columns 3, 4, and 5 show the performance of the models on that image

homogeneous illumination of images, where conventional methods usually tend to obtain unsatisfactory results. The development of accurate automatic tools using CNNs would reduce the time required for xylem quantitative anatomy, as well as overcoming of the need of acquiring proprietary software. A reduction of entry barriers associated with quantitative xylem anatomy may serve to widen the community using this technique as well as the ecological problems approached. In this sense, as a future work, our aim is to develop a user-friendly tool for these models providing a base segmentation from which possible errors can be corrected interactively. Moreover, the existence of a community using this tool would lead to an iterative improvement in the strength of the algorithm.

Acknowledgements Alex Fajardo collected branch sections. This work has been developed within SEÑALES (VA026P17) Project funded by Junta de Castilla y León and FEDER. We gratefully acknowledge the support of NVIDIA Corporation with the donation of the Titan Xp GPU used for this research. A. G.-C. was supported by a Juan de la Cierva-Formación Grant (FJCI-2015- 24770) from the Spanish Ministry of Economy and Competitiveness. M. L.-S. thanks the Centro de Recursos Hídricos para la Agricultura y la Minería CRHIAM (CONICYT-FONDAP-1513001).

\section{Compliance with ethical standards}

Conflict of interest The authors declare no conflict of interest.

\section{References}

1. Malhi Y, Meir P, Brown S (2002) Forests, carbon and global climate. Philos Trans R Soc Lond Ser A Math Phys Eng Sci 360(1797):1567-1591

2. Cleland EE, Chuine I, Menzel A, Mooney HA, Schwartz MD (2007) Shifting plant phenology in response to global change. Trends Ecol Evol 22(7):357-365

3. Choat B, Brodribb TJ, Brodersen CR, Duursma RA, López R, Medlyn BE (2018) Triggers of tree mortality under drought. Nature 558(7711):531

4. Fonti P, von Arx G, García-González I, Eilmann B, Sass-Klaassen U, Gärtner H, Eckstein D (2010) Studying global change through investigation of the plastic responses of xylem anatomy in tree rings. New Phytol 185(1):42-53

5. Selig B, Luengo H, Bardage S, Borgefors G (2009) Segmentation of highly lignified zones in wood fiber cross-sections. Lecture 
notes in computer science (including subseries lecture notes in artificial intelligence and lecture notes in bioinformatics), vol 5575. LNCS, pp 369-378

6. Hacke UG, Spicer R, Schreiber SG, Plavcová L (2017) An ecophysiological and developmental perspective on variation in vessel diameter. Plant Cell Environ 40(6):831-845

7. García-Cervigón AI, Fajardo A, Caetano-Sánchez C, Camarero JJ, Olano JM. Xylem anatomy needs to change, so that conductivity can stay the same: xylem adjustments across elevation and latitude in Nothofagus pumilio

8. von Arx G, Carrer M (2014) Roxas-a new tool to build centuries-long tracheid-lumen chronologies in conifers. Dendrochronologia 32(3):290-293. https://doi.org/10.1016/j.dendro. 2013.12.001

9. Olano JM, Eugenio M, García-Cervigón AI, Folch M, Rozas V (2012) Quantitative tracheid anatomy reveals a complex environmental control of wood structure in continental mediterranean climate. Int J Plant Sci 173(2):137-149

10. Speer JH (2010) Fundamentals of tree-ring research. University of Arizona Press, Tucson

11. Gärtner $\mathrm{H}$, Cherubini $\mathrm{P}$, Fonti $\mathrm{P}$, von Arx G, Schneider L, Nievergelt D, Verstege A, Bast A, Schweingruber FH, Büntgen U (2015) A technical perspective in modern tree-ring research-how to overcome dendroecological and wood anatomical challenges. J Vis Exp JoVE 5(97):e52337

12. Arx GV, Dietz H (2005) Automated image analysis of annual rings in the roots of perennial forbs. Int $\mathrm{J}$ Plant Sci 166(5):723-732

13. Meijering E (2012) Cell segmentation: 50 years down the road [life sciences]. IEEE Signal Process Mag 29(5):140-145. https:// doi.org/10.1109/MSP.2012.2204190

14. Moëll M, Donaldson L (2001) Comparison of segmentation methods for digital image analysis of confocal microscope images to measure tracheid cell dimensions. IAWA J 22(3):267-288. https://doi.org/10.1163/22941932-90000284

15. Land A, Wehr M, Roelfs KU, Epkes S, Reichle D, Kauer G (2017) A novel computer-aided tree-ring analysis software (CATS): oak earlywood vessel size reveals a clear spring heat sum response. Trees 31(5):1683-1695. https://doi.org/10.1007/ s00468-017-1578-7

16. Nedzved A, Mitrović AL, Savić A, Mutavdžić D, Radosavljević JS, Pristov JB, Steinbach G, Garab G, Starovoytov V, Radotić K (2018) Automatic image processing morphometric method for the analysis of tracheid double wall thickness tested on juvenile picea omorika trees exposed to static bending. Trees 32(5):1347-1356

17. Chopin J, Laga H, Huang CY, Heuer S, Miklavcic SJ (2015) RootAnalyzer: a cross-section image analysis tool for automated characterization of root cells and tissues. PLoS ONE 10(9):e0137655. https://doi.org/10.1371/journal.pone.0137655

18. Wang H, Qi H, Li W, Zhang G, Wang P (2009) A GA-based automatic pore segmentation algorithm. Assoc Computing Machinery, New York

19. Wang H, Zhang G, Qi H, Ma L (2009) Multi-objective optimization on pore segmentation. In: 2009 fifth international conference on natural computation, vol 4, pp 613-617. https://doi. org/10.1109/ICNC.2009.572

20. Zhang S, Xu W, Meng Z (2010) Study on method to dissected data of wood cell image. Adv Mater Res 139-141:303-307. https://doi.org/10.4028/www.scientific.net/AMR.139-141.303

21. Mallik A, Tarrio-Saavedra J, Francisco-Fernandez M, Naya S (2011) Classification of wood micrographs by image segmentation. Chemom Intell Lab Syst 107(2):351-362. https://doi.org/10. 1016/j.chemolab.2011.05.005

22. Guang-Sheng C, Peng Z (2013) Wood cell recognition using geodesic active contour and principal component analysis. Opt
Int J Light Electron Opt 124(10):949-952. https://doi.org/10. 1016/j.ijleo.2012.02.032

23. Qi HN, Chen FN, Ma LF (2007) Pore feature segmentation based on mathematical morphology. In: IECON 2007-33rd annual conference of the IEEE industrial electronics society, pp 2474-2477. https://doi.org/10.1109/IECON.2007.4460248

24. Pan S, Kudo M (2011) Segmentation of pores in wood microscopic images based on mathematical morphology with a variable structuring element. Comput Electron Agric 75(2):250-260. https://doi.org/10.1016/j.compag.2010.11.010

25. Wunderling A, Ben Targem M, Barbier de Reuille P, Ragni L (2016) Novel tools for quantifying secondary growth. J Exp Bot 68(1):89-95

26. Hall HC, Fakhrzadeh A, Luengo Hendriks CL, Fischer U (2016) Precision automation of cell type classification and sub-cellular fluorescence quantification from laser scanning confocal images. Front Plant Sci 7:119

27. Travis A, Hirst D, Chesson A (1996) Automatic classification of plant cells according to tissue type using anatomical features obtained by the distance transform. Ann Bot 78(3):325-331

28. Brunel G, Borianne P, Subsol G, Jaeger M, Caraglio Y (2012) Automatic characterization of the cell organization in light microscopic images of wood: application to the identification of the cell file. In: 2012 IEEE 4th international symposium on plant growth modeling, simulation, visualization and applications, pp 58-65. https://doi.org/10.1109/PMA.2012.6524813

29. Brunel G, Borianne P, Subsol G, Jaeger M, Caraglio Y (2014) Automatic identification and characterization of radial files in light microscopy images of wood. Ann Bot 114(4):829-840. https://doi.org/10.1093/aob/mcu119

30. Kennel P, Subsol G, Gueroult M, Guéroult M, Borianne P (2010) Automatic identification of cell files in light microscopic images of conifer wood. In: 2010 2nd international conference on image processing theory, tools and applications, pp 98-103. https://doi. org/10.1109/IPTA.2010.5586800

31. Guan X, Sun L, Cao J (2006) Level set method based on improved Mumford-Shah model applied in wood cell image segmentation. In: The 2006 IEEE international joint conference on neural network proceedings, pp 2315-2318. https://doi.org/10. 1109/IJCNN.2006.247031

32. Zhao L, Ma Y (2010) Wood adhesion cell segmentation scheme based on GVF-Snake model. In: International conference on image processing and pattern recognition in industrial engineering, vol 7820, p 78200N. International society for optics and photonics. https://doi.org/10.1117/12.866337

33. Fernando Espinosa L, Javier Herrera R, Polanco-Tapia C (2015) Segmentation of anatomical elements in wood microscopic images using artificial vision techniques. Maderas-Ciencia Y Tecnol 17(4):735-748. https://doi.org/10.4067/S0718221X2015005000064

34. Roncancio HA, Velasco HF, Herrera RJ (2003) Segmentation of wood microanatomy images using multiscale classification. In: Proceedings of the 3rd IEEE international symposium on signal processing and information technology (IEEE Cat. No.03EX795), pp 692-695. https://doi.org/10.1109/ISSPIT.2003.1341215

35. García-Pedrero A, Gonzalo-Martín C, Lillo-Saavedra M (2017) A machine learning approach for agricultural parcel delineation through agglomerative segmentation. Int $\mathbf{J}$ Remote Sens 38(7):1809-1819. https://doi.org/10.1080/01431161.2016. 1278312

36. Wu W, Chen AY, Zhao L, Corso JJ (2014) Brain tumor detection and segmentation in a crf (conditional random fields) framework with pixel-pairwise affinity and superpixel-level features. Int $\mathbf{J}$ Comput Assist Radiol Surg 9(2):241-253 
37. Ma B, Ban X, Huang H, Chen Y, Liu W, Zhi Y (2018) Deep learning-based image segmentation for Al-La Alloy microscopic images. Symmetry 10(4):107

38. Fu H, Xu Y, Lin S, Wong DWK, Liu J (2016) Deepvessel: retinal vessel segmentation via deep learning and conditional random field. In: International conference on medical image computing and computer-assisted intervention, pp 132-139. Springer

39. Van Valen DA, Kudo T, Lane KM, Macklin DN, Quach NT, DeFelice MM, Maayan I, Tanouchi Y, Ashley EA, Covert MW (2016) Deep learning automates the quantitative analysis of individual cells in live-cell imaging experiments. PLoS Comput Biol 12(11):e1005177

40. Yao Z, Zhang Z, Xu LQ (2016) Convolutional neural network for retinal blood vessel segmentation. In: 2016 9th international symposium on Computational intelligence and design (ISCID), vol 1. IEEE, pp 406-409

41. Ronneberger O, Fischer P, Brox T (2015) U-net: convolutional networks for biomedical image segmentation. In: International conference on medical image computing and computer-assisted intervention. Springer, pp 234-241

42. Biem A (2014) Neural networks: a review. In: Aggarwal CC (ed) Data classification: algorithms and applications. CRC press, pp 205-244

43. Garcia-Pedrero A, García-Cervigón A, Caetano C, CalderónRamírez S, Olano JM, Gonzalo-Martín C, Lillo-Saavedra M, García-Hidalgo M (2018) Xylem vessels segmentation through a deep learning approach: a first look. In: 2018 IEEE international work conference on bioinspired intelligence (IWOBI), pp 1-9. https://doi.org/10.1109/IWOBI.2018.8464184

44. Den Bakker I (2017) Python deep learning cookbook: over 75 practical recipes on neural network modeling, reinforcement learning, and transfer learning using Python. Packt Publishing Ltd, Birmingham

45. Dettmann S, Pérez CA, Thomas FM (2013) Xylem anatomy and calculated hydraulic conductance of four Nothofagus species with contrasting distribution in South-Central Chile. Trees 27(3):685-696

46. Körner C (2003) Alpine plant life: functional plant ecology of high mountain ecosystems; with 47 tables. Springer, Berlin
47. Gärtner H, Lucchinetti S, Schweingruber F (2015) A new sledge microtome to combine wood anatomy and tree-ring ecology. IAWA J 36(4):452-459

48. Iglovikov V, Mushinskiy S, Osin V (2017) Satellite imagery feature detection using deep convolutional neural network: a kaggle competition. arXiv preprint arXiv:1706.06169

49. Chaurasia A, Culurciello E (2017) Linknet: exploiting encoder representations for efficient semantic segmentation. In: 2017 IEEE visual communications and image processing (VCIP). IEEE, pp 1-4

50. Lin TY, Dollár P, Girshick R, He K, Hariharan B, Belongie S (2017) Feature pyramid networks for object detection. In: Proceedings of the IEEE conference on computer vision and pattern recognition, pp 2117-2125

51. He K, Zhang X, Ren S, Sun J (2016) Deep residual learning for image recognition. In: Proceedings of the IEEE conference on computer vision and pattern recognition, pp 770-778

52. Goodfellow I, Bengio Y, Courville A (2016) Deep learning. MIT Press. http://www.deeplearningbook.org

53. Iglovikov V, Shvets A (2018) Ternausnet: U-net with VGG11 encoder pre-trained on imagenet for image segmentation. arXiv preprint arXiv: 1801.05746

54. He K, Girshick R, Dollár P (2018) Rethinking imagenet pretraining. arXiv preprint arXiv: 1811.08883

55. Chollet $\mathrm{F}$ et al (2015) Keras. https://keras.io

56. Moolayil J (2018) Learn keras for deep neural networks: a fasttrack approach to modern deep learning with Python, 1st edn. Apress, Berkely

57. Rahman MA, Wang Y (2016) Optimizing intersection-over-union in deep neural networks for image segmentation. In: International symposium on visual computing. Springer, pp 234-244

58. Garcia-Garcia A, Orts-Escolano S, Oprea S, Villena-Martinez V, Garcia-Rodriguez J (2017) A review on deep learning techniques applied to semantic segmentation. arXiv preprint arXiv:1704. 06857

Publisher's Note Springer Nature remains neutral with regard to jurisdictional claims in published maps and institutional affiliations. 